\title{
Comparison of Two Forms of Loperamide-Simeticone and a Probiotic Yeast (Saccharomyces boulardii) in the Treatment of Acute Diarrhoea in Adults: A Randomised Non-Inferiority Clinical Trial
}

\author{
Jeremy Cottrell ${ }^{1} \cdot$ Kerstin Koenig $^{1} \cdot$ Roland Perfekt $^{2} \cdot$ Robert Hofmann $^{3}$. \\ For the Loperamide-Simethicone Acute Diarrhoea Study Team
}

Published online: 5 November 2015

(c) The Author(s) 2015. This article is published with open access at Springerlink.com

\begin{abstract}
Background Acute diarrhoea is a frequent health problem in both travellers and residents that has a social and economic impact. This study compared the efficacy and tolerability of two loperamide-simeticone formulations and a Saccharomyces boulardii capsule as symptomatic treatment.

Methods This was a prospective, randomised, single (investigator)-blind, three-arm, parallel group, non-inferiority clinical trial in adult subjects with acute diarrhoea at clinics in Mexico and India, with allocation to a loperamide-simeticone $2 / 125 \mathrm{mg}$ caplet or chewable tablet (maximum eight in $48 \mathrm{~h}$ ) or $S$. boulardii ( $250 \mathrm{mg}$ twice daily for 5 days).

Outcome Measures The primary outcome measure was the number of unformed stools between 0 and $24 \mathrm{~h}$ following the initial dose of study medication (NUS 0-24). The secondary outcome measures were time to last unformed stool (TLUS), time to complete relief of

This study has been previously presented at a scientific meeting: Clinical study comparing loperamide-simeticone and the probiotic Saccharomyces boulardii in acute diarrhoea. United European Gastroenterology (UEG) Week; 23-27 October 2010; Barcelona, Spain.
\end{abstract}

The members of the Loperamide-Simethicone Acute Diarrhoea Study Team are listed in Acknowledgments.

Jeremy Cottrell

jcottre1@its.jnj.com

1 Medical Affairs, Johnson \& Johnson Ltd, Foundation Park, Roxborough Way, Maidenhead SL6 3UG, UK

2 McNeil AB, PO Box 941, 25109 Helsingborg, Sweden

3 Johnson \& Johnson GmbH, Johnson \& Johnson Platz 2 (Raiffeisenstraße 9), 41470 Neuss, Germany diarrhoea (TCRD), time to complete relief of abdominal discomfort (TCRAD) and the subject's evaluation of treatment effectiveness. Follow-up endpoints at 7 days were feeling of complete wellness; stool passed since final study visit; and continued or recurrent diarrhoea.

Subjects In this study, 415 subjects were randomised to either a loperamide-simeticone caplet $(n=139)$, loperamide-simeticone chewable tablet $(n=139)$ or $S$. boulardii capsule $(n=137)$ and were included in the intention-to-treat analysis.

Results With regards to mean NUS 0-24, the loperamide-simeticone caplet was non-inferior to loperamide-simeticone tablets (3.4 vs. 3.3; one-sided $97.5 \%$ confidence interval $\leq 0.5$ ), with both significantly lower than $S$. boulardii $(4.3 ; p<0.001)$. The loperamidesimeticone groups had a shorter median TLUS [14.9 and 14.0 vs. $28.5 \mathrm{~h}$ (loperamide-simeticone caplet and chewable tablet groups, respectively, vs. $S$. boulardii); $p<0.001$ ], TCRD (26.0 and 26.0 vs. $45.8 \mathrm{~h} ; p<0.001$ ) and TCRAD (12.2 and 12.0 vs. $23.9 \mathrm{~h} ; p<0.005)$ than $S$. boulardii. Treatment effectiveness for overall illness, diarrhoea and abdominal discomfort relief was greater $(p<0.001)$ in the loperamide-simeticone groups than with $S$. boulardii. At 7-day follow-up most subjects reported passing stool at least once since the final study visit (loperamide-simeticone caplet $94.1 \%$, loperamide-simeticone chewable tablet $94.8 \%$, S. boulardii $97.0 \%$ ), did not experience continued or recurrent diarrhoea [loperamidesimeticone caplet $3.7 \%$ ( $p<0.03$ vs. S. boulardii), loperamide-simeticone chewable tablet $3.7 \%$, S. boulardii $5.7 \%$ ] and felt completely well [loperamide-simeticone caplet $96.3 \%(p<0.02$ vs. S. boulardii), loperamidesimeticone chewable tablet $96.3 \% \quad(p<0.02 \quad$ vs. $S$. boulardii), S. boulardii $88.6 \%$ ]. All treatments were well-tolerated with few adverse events. 
Conclusions The loperamide-simeticone caplet was noninferior to the original loperamide-simeticone chewable tablet formulation; both formulations can be expected to demonstrate similar clinical efficacy in the relief of symptoms of acute diarrhoea. Both loperamide-simeticone formulations were superior to the $S$. boulardii capsule in the primary and secondary endpoints.

Clinical Trial Registration ClinicalTrials.gov identifier NCT00807326.

\section{Key Points}

The loperamide-simeticone caplet formulation was demonstrated to be non-inferior to the original chewable tablet formulation; both formulations can be expected to demonstrate similar clinical efficacy in the relief of symptoms of acute diarrhoea in adults.

The Saccharomyces boulardii capsule used at the approved dose was significantly less effective than the loperamide-simeticone formulations in relieving the symptoms of acute diarrhoea in adults.

$S$. boulardii may have more of a role in diarrhoea in children, more persistent diarrhoea or diarrhoea prevention.

\section{Introduction}

Acute diarrhoea is a common diagnosis in general practice, although most affected individuals do not consult a physician. It has been estimated that the annual rate of acute diarrhoea in industrialised countries averages 0.5-2 episodes per person per year [1]. Many episodes of diarrhoea are travel-related; traveller's diarrhoea (TD) has an attack rate from 20 to over $60 \%$ depending on the destination [2], and it has been estimated that the illness affects 15-20 million people annually [3]. Although in most cases self-limiting, TD causes substantial disruption by interfering with travel itineraries, business opportunities and tourist industry revenues. Acute uncomplicated diarrhoea is commonly managed in travellers and residents alike with adequate hydration and self-medication.

Loperamide is an effective antidiarrhoeal agent with antimotility and antisecretory actions, and is the preferred symptomatic treatment for diarrhoea for most non-febrile, non-dysenteric cases [4-7]. A loperamide-simeticone combination chewable tablet is widely available and is indicated for the symptomatic treatment of acute diarrhoea associated with gas-related abdominal discomfort, including bloating, cramping or flatulence. Two doubleblind, randomised placebo-controlled studies demonstrated that a loperamide-simeticone combination chewable tablet was significantly more effective in the relief of acute diarrhoea than either loperamide or simeticone alone or placebo over a 48-h study period [8,9]. Both studies were conducted in Mexico and included mixed populations of international travellers and local residents. These studies suggest that loperamide and simeticone may act synergistically to enhance efficacy in acute diarrhoea, possibly explained by modified intestinal kinetics of loperamide when formulated with simeticone [10]. A loperamidesimeticone caplet (capsule-shaped tablet) formulation was subsequently developed, but there are no clinical data on the efficacy of this formulation. More recently, a comparison of loperamide-simeticone with loperamide alone using a mannitol model of secretory diarrhoea demonstrated that both forms of loperamide significantly reduced small bowel water content, but that the loperamidesimeticone combination also significantly reduced ascending colon water content [11]. This finding is consistent with the greater clinical efficacy found with the combination, although a higher than usual dose was used in this study.

Saccharomyces boulardii is a non-pathogenic probiotic yeast used first in France to treat diarrhoea in the beginning of the 1950s, and is now widely available worldwide in a lyophilised form. The antidiarrhoeal effect of lyophilised $S$. boulardii has been investigated in various forms of diarrhoeal diseases, including antibacterial-associated and acute infectious diarrhoea in adults and children, with evidence of efficacy [12-21]. Although the exact mechanism by which $S$. boulardii might exert its activity remains unclear, several possible mechanisms have been proposed, including inhibition of pathogen adhesion, strengthening of enterocyte tight junctions, neutralisation of bacterial virulence factors and enhancement of the mucosal immune response [13].

The aim of this study was to compare the efficacy and safety of two formulations of loperamide-simeticone combination in the treatment of acute diarrhoea in adults, on the basis of a therapeutic non-inferiority design; the study also compares the loperamide-simeticone combinations versus a $S$. boulardii probiotic capsule.

\section{Patients and Methods}

\subsection{Study Design}

This was a prospective, randomised, single (investigator)blind, three-arm, parallel group, non-inferiority clinical trial conducted in accordance with the principles of the 1964 Declaration of Helsinki and later amendments, and 
with applicable Good Clinical Practice (GCP) guidelines. Local regulatory requirements were followed, and the study protocol and associated documents were reviewed and approved by the Ethics Committees for each participating site. The study was registered on ClinicalTrials.gov (NCT00807326).

\subsection{Participants}

Four clinical sites in Mexico and two in Goa, India participated in the study. Publicity posters and cards were used to raise awareness of the study among both travellers and local residents. Potentially eligible subjects were invited to attend either outreach clinics or the investigator sites for assessment. Male or female subjects aged 18 years or over were recruited if they had symptoms of acute diarrhoeal illness with onset during the prior $48 \mathrm{~h}$, a minimum of three unformed stools in the $24 \mathrm{~h}$ before study entry with the most recent stool unformed, and with abdominal discomfort within the prior $4 \mathrm{~h}$. All subjects provided written informed consent prior to inclusion in the study.

Exclusion criteria were requiring hospital admission, parenteral hydration or antibacterial therapy; axillary temperature $>38.2{ }^{\circ} \mathrm{C}$ or oral temperature $>38.6{ }^{\circ} \mathrm{C}$; history or clinical evidence of gross blood or pus in stool; orthostatic hypotension; unable to take medication and fluids by mouth; chronic gastrointestinal disease, hepatic or renal insufficiency, or other significant medical condition; immunodeficiency; antibacterials, oral antifungals, quinidine or ritonavir within 7 days, antidiarrhoeal or prokinetic drugs, antiflatulents, probiotics or bismuth salts within $48 \mathrm{~h}$, or any analgesic within $6 \mathrm{~h}$ prior to study entry; use of opiates; hypersensitivity to study medication; pregnant or breast-feeding; and unable to comply with the study requirements.

\subsection{Intervention}

Eligible subjects providing informed consent were sequentially assigned to study medication according to a randomised schedule. The subjects were provided with either:

(A) Loperamide-simeticone $2 / 125 \mathrm{mg}$ capsule-shaped tablet (caplet), in total eight caplets (Imodium ${ }^{\circledR}$ Plus Caplet, McNeil Products Ltd., Maidenhead, UK); or

(B) Loperamide-simeticone $2 / 125 \mathrm{mg}$ chewable tablets, in total eight tablets (Imodium $^{\circledR}$ Plus chewable tablet, McNeil Products Ltd., Maidenhead, UK); or

(C) S. boulardii $250 \mathrm{mg}$ capsules, in total ten capsules (Perenterol $^{\circledR} \quad$ Forte $250 \mathrm{mg}$ capsules, MEDICE Arzneimittel Pütter GmbH \& Co. KG. Iserlohn, Germany).
For treatments A and B, two caplets or chewable tablets were taken initially at the investigator site, followed subsequently by one caplet/tablet after each unformed stool, with a maximum of four caplets/tablets in a $24-\mathrm{h}$ period, for up to $48 \mathrm{~h}$. For treatment $\mathrm{C}$, subjects were asked to take one capsule twice a day for 5 days.

Subjects recorded their symptoms, time and consistency of bowel movements, and time of study medication administration for the 48 -h period after their initial dose in a study diary. Stool consistency was rated according to a 3-point scale of formed, soft or liquid. Subjects rated the intensity of their gas-related abdominal discomfort from 0 (absent) to 4 (severe) hourly over the first $4 \mathrm{~h}$ of the study, and at $8,12,18,24,36$ and $48 \mathrm{~h}$ after the initial dose. At 12,24 or $48 \mathrm{~h}$ or at the time of discontinuation from the study, subjects recorded if and when they experienced complete relief from their diarrhoea. At $48 \mathrm{~h}$ or at the time of discontinuation from the study, subjects recorded an overall evaluation of treatment effectiveness, as well as overall evaluations of the treatment effectiveness in relieving gas-related abdominal discomfort and diarrhoea (separately) on a 5 -point scale ranging from 0 (poor) to 4 (excellent). Adverse events were recorded throughout the study using patient diaries.

After $48 \mathrm{~h}$ in the study and within $72 \mathrm{~h}$ of entry, each patient returned to the study site for a second visit, at which time the patient diary was returned; at this visit, the entire diary was reviewed with the patient to ensure that all required information had been recorded and any reported adverse events were noted.

Subjects were issued with cards for follow-up questions at 7 days after baseline and asked whether they would prefer to respond by return of the card, email or telephone interview. Subjects were asked the following questions: "Have you passed stool since your final study visit?"; "Has your diarrhoea continued or returned since your final study visit?"; "If diarrhoea has continued or returned, how many unformed (soft or liquid) stool have you passed since your final study visit?"; and "Do you feel completely well following your diarrhoea illness?".

\subsection{Endpoints}

The primary endpoint was the number of unformed stools passed between 0 and $24 \mathrm{~h}$ following the initial dose of study medication (NUS 0-24).

Secondary endpoints included time to last unformed stool (TLUS), time to complete relief of diarrhoea (TCRD), time to complete relief of abdominal discomfort (TCRAD) and subject's evaluation of treatment effectiveness for overall illness relief, diarrhoea relief and abdominal 
discomfort relief, and number and severity of reported adverse events during the study.

The follow-up endpoints at 7 days were feeling completely well following diarrhoea illness; stool passed at least once between study visit 2 and follow-up; and continued or recurrent diarrhoea between final study visit and follow-up.

\subsection{Sample Size}

Sample size estimation was based on NUS 0-24, with an assumed standard deviation of 1.3 , no expected mean treatment difference for the two loperamide-simeticone groups and a pre-specified non-inferiority margin of 0.5 . If the number of evaluable primary endpoint data in each group was 108, a two-group one-sided $t$-test of significance level of 0.025 aiming to show non-inferiority of caplets relative to tablets had $80 \%$ power to reject the null hypothesis that the mean treatment difference (caplets minus tablets) in NUS 0-24 was greater than the non-inferiority margin of 0.5 versus the alternative hypothesis that the mean treatment difference was $\leq 0.5$. Allowing for drop-outs and for non-evaluable data, a minimum sample size of 125 per group was required.

\subsection{Randomisation and Blinding}

Subjects were randomly assigned to one of the three treatment groups based on a computer-generated randomisation schedule prepared by the sponsor before the study. Based on this schedule, the study drug was packaged and labelled for each subject. Subject randomisation numbers were pre-printed on the labels and assigned sequentially as subjects were recruited. The randomisation was stratified by investigator site, achieved by issuing blocks of consecutive numbers to each site.

The differing nature of the dose forms, with divergent posology, did not permit a full double-blind design. A single-blinded approach (with investigators blind to treatment group) was adopted to minimise potential bias during data collection and evaluation of clinical endpoints. Study medication was presented in sealed cartons of identical appearance, differing only in the allocation code. Each recruited subject was sequentially allocated the next available number.

To maintain the blind, sealed envelopes containing the study drug identification were provided to the investigator; these were not used in the course of the study. The allocation code was broken only after all subjects had completed the study and the database had been locked in order to perform statistical analysis. Administration of the study medication was supervised at the site by clinical personnel not otherwise connected with the study.

\subsection{Statistical Methods}

All output was produced using Statistical Analysis Software $\left(\mathrm{SAS}^{\circledR}\right.$ ) version 9.1.3 (SAS Institute Inc., Cary, NC, USA) combined with AdClin $^{\circledR}$ (AdClin S.A., Paris, France).

The test for non-inferiority (NUS 0-24) of loperamidesimeticone caplets versus tablets was one-sided with $\alpha$ equal to 0.025 ; all other tests were two-sided at a 0.05 level.

The efficacy analysis was performed using an intentionto-treat (ITT) set, which included all randomised subjects, with imputation for missing efficacy endpoint data. A last observation carried forward (LOCF) approach was used for the primary endpoint (NUS 0-24), in this case the baseline values; for the time-to-event analyses, missing values were right censored at $48 \mathrm{~h}$; and for subject evaluation of treatment effectiveness, missing data were replaced with the most negative outcome of 'poor'. For the follow-up endpoints at 7 days, an efficacy set based on all patients completed was used, with no imputation of data.

An analysis of variance (ANOVA) with effects for treatment (either loperamide-simeticone caplets or chewable tablets) and investigator site was used to derive a onesided $97.5 \%$ confidence interval (CI), bounded from above, for the mean difference between loperamidesimeticone caplets and chewable tablets with respect to the primary endpoint (NUS 0-24). Non-inferiority was concluded if the (upper) bound of the one-sided $97.5 \% \mathrm{CI}$ was $\leq 0.5$, the non-inferiority margin. An ANOVA with effects for treatment and investigator site was used to construct two-sided $95 \%$ CIs for the mean differences between the $S$. boulardii group and loperamide-simeticone caplet and chewable tablet groups with respect to NUS 0-24.

Time-to-event analyses (TLUS, TCRD, TCRAD) were performed pairwise by means of the log-rank test stratified for investigator site.

For subject's evaluation of treatment effectiveness for overall illness relief, diarrhoea relief and abdominal discomfort relief, frequency distributions were compared pairwise between the three treatment groups using the Chisquare $\left(\chi^{2}\right)$ test or Fisher's exact test. For the follow-up endpoints at 7 days, frequency distributions between the three treatment groups were compared pairwise using a $\chi^{2}$ test (or Fisher's exact test).

\section{Results}

Figure 1 shows the flow of subjects through the study. The first subject's first visit was on 12 November 2008 and the last subject's last visit took place on 30 August 2009. The demographic (Table 1) and clinical characteristics 


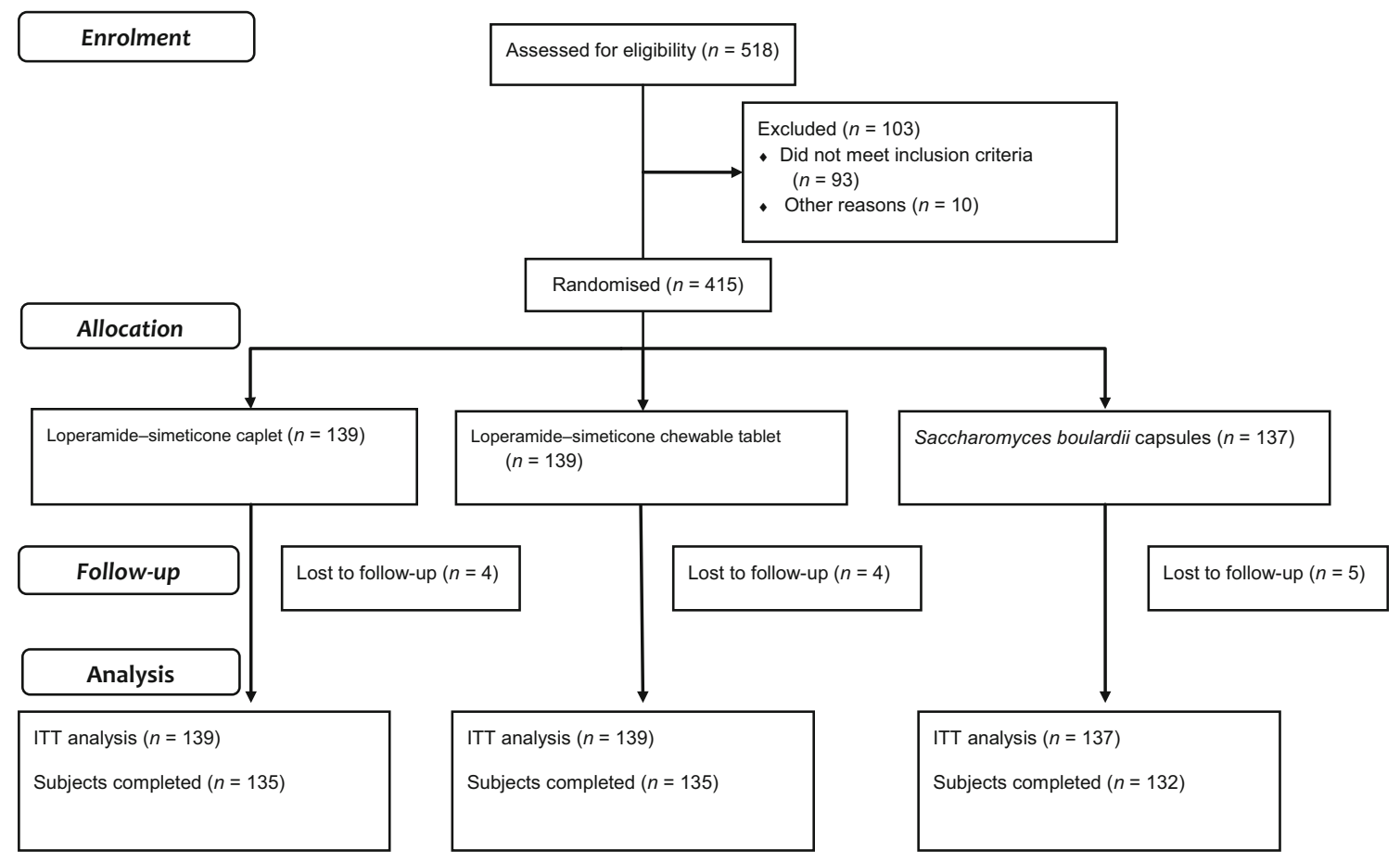

Fig. 1 Study flow diagram. ITT intention-to-treat

Table 1 Demographics

\begin{tabular}{lllll}
\hline Demographic & $\begin{array}{l}\text { Loperamide- } \\
\text { simeticone caplet }\end{array}$ & $\begin{array}{l}\text { Loperamide-simeticone } \\
\text { chewable tablet }\end{array}$ & $\begin{array}{l}\text { Saccharomyces } \\
\text { boulardii capsule }\end{array}$ & Total \\
\hline$N$ & 139 & 139 & 137 & 415 \\
Age (years) & & & 36.9 & 36.4 \\
Mean & 36.2 & 36.1 & $19-79$ & $18-79$ \\
Range & $19-72$ & $18-72$ & & \\
Sex $[n(\%)]$ & & & $93(68)$ & $267(64)$ \\
Male & $93(67)$ & $81(58)$ & $44(32)$ & $148(36)$ \\
Female & $46(33)$ & $58(42)$ & $37(27)$ & $114(27)$ \\
Country/region of origin $[n(\%)]$ & & $37(27)$ & $96(23)$ \\
Mexico & $44(32)$ & $33(24)$ & $27(20)$ & $82(20)$ \\
USA/Canada & $26(19)$ & $33(24)$ & $20(15)$ & $72(17)$ \\
India & $23(17)$ & $32(23)$ & $13(9)$ & $30(7)$ \\
Europe & $26(19)$ & $26(19)$ & $2(1)$ & $10(2)$ \\
Russia & $11(8)$ & $6(4)$ & $0(0)$ & $11(3)$ \\
South America & $4(3)$ & $4(3)$ & & \\
Other & $5(4)$ & $5(4)$ & & \\
\hline
\end{tabular}

(Table 2) of the subjects enrolled in the three groups were comparable at baseline. Similar proportions of travellers and local residents participated: $47 \%$ of subjects were from Mexico or India, with most international travellers to these countries originating from either North America (23\%) or Europe $(17 \%)$. The ITT set consisted of 415 $(100 \%)$ subjects.

\subsection{Primary Endpoint}

\subsubsection{Number of Unformed Stools (0-24 h)}

The primary endpoint was NUS 0-24 following the initial dose of study medication; results are presented in Table 3. The mean values were similar between the 
Table 2 Clinical characteristics at baseline

\begin{tabular}{|c|c|c|c|c|}
\hline Characteristic & $\begin{array}{l}\text { Loperamide- } \\
\text { simeticone } \\
\text { caplet }\end{array}$ & $\begin{array}{l}\text { Loperamide- } \\
\text { simeticone } \\
\text { chewable tablet }\end{array}$ & $\begin{array}{l}\text { Saccharomyces } \\
\text { boulardii } \\
\text { capsule }\end{array}$ & Total \\
\hline$N$ & 139 & 139 & 137 & 415 \\
\hline $\begin{array}{l}\text { Duration of diarrhoeal illness [mean } \\
\text { (h)] }\end{array}$ & 22.2 & 21.9 & 22.7 & 22.3 \\
\hline Time since last stool $[\text { mean }(\mathrm{h})]^{\mathrm{a}}$ & 1.9 & 1.9 & 2.5 & 2.1 \\
\hline \multicolumn{5}{|l|}{ Consistency of last stool $[n(\%)]^{\mathrm{b}}$} \\
\hline Grade 2 & $33(24)$ & $42(30)$ & $38(28)$ & $113(27)$ \\
\hline Grade 3 & $106(76)$ & $97(70)$ & $99(72)$ & $302(73)$ \\
\hline $\begin{array}{l}\text { Mean number of unformed stools in the } \\
\text { past } 24 \mathrm{~h}\end{array}$ & 5.9 & 5.4 & 5.5 & 5.6 \\
\hline Severe abdominal discomfort $[n(\%)]^{\mathrm{c}}$ & $55(40)$ & $48(34)$ & $48(35)$ & $151(36)$ \\
\hline \multicolumn{5}{|c|}{${ }^{\mathrm{a}}$ Calculated to time of first dose of study medication } \\
\hline
\end{tabular}

loperamide-simeticone caplet group (3.4) and the chewable tablet group (3.3). The (upper) limit of the onesided $97.5 \%$ CI for the difference (caplets - tablets) was 0.50 , equal to the predetermined non-inferiority margin, and therefore non-inferiority was concluded. Both loperamide-simeticone groups had a significantly lower mean NUS 0-24 than the $S$. boulardii capsule group (4.3) (both $p<0.001$ ).

Analysis of the primary endpoint based on all subjects completed without imputation $(n=403)$ gave a similar outcome. In this analysis, mean NUS 0-24 values were 3.3 in the loperamide-simeticone caplet group, 3.2 in the chewable tablet group and 4.3 in the $S$. boulardii group. The (upper) limit of the one-sided $97.5 \%$ CI for the difference (caplets - tablets) was 0.48 , within the predetermined non-inferiority margin.

\subsection{Secondary Endpoints}

\subsubsection{Time to Last Unformed Stool}

The estimated median TLUS (Kaplan-Meier analysis) was significantly longer in the $S$. boulardii capsule group $(28.5 \mathrm{~h})$ than in either the loperamide-simeticone caplet $(14.9 \mathrm{~h})$ or chewable tablet $(14.0 \mathrm{~h})$ groups. There was no significant difference between loperamide-simeticone caplets and chewable tablets, while the differences between both loperamide-simeticone caplets and chewable tablets and $S$. boulardii capsule were significant $(p<0.001)$. The Kaplan-Meier curve for the TLUS is shown in Fig. 2.

\subsubsection{Time to Complete Relief of Diarrhoea}

Overall, the median TCRD (Kaplan-Meier analysis) was significantly longer in the $S$. boulardii capsule group $(45.8 \mathrm{~h})$ than in both loperamide-simeticone groups (both $26.0 \mathrm{~h} ; p<0.001)$. The loperamide-simeticone caplets and tablets groups were not significantly different. Figure 3 shows the Kaplan-Meier curve for the TCRD.

\subsubsection{Time to Complete Relief of Abdominal Discomfort}

Overall, the median TCRAD (Kaplan-Meier analysis) was significantly longer in the $S$. boulardii capsule group $(23.9 \mathrm{~h})$ than in the loperamide-simeticone caplet and chewable tablet groups (12.2 and $12.0 \mathrm{~h}$, respectively; $p<0.005)$. No significant difference between loperamidesimeticone caplets and chewable tablets was found.

\subsubsection{Subject's Evaluation of Treatment Effectiveness}

The proportion of subjects evaluating treatment effectiveness as 'very good' or 'excellent' was higher in both the loperamide-simeticone caplet group $(83.5 \%)$ and chewable tablet group $(85.6 \%)$ than in the $S$. boulardii capsule group $(48.9 \%)$ for overall illness relief, higher in the loperamide-simeticone caplet group $(79.9 \%)$ and chewable tablet group (84.9\%) than in the S. boulardii capsule group $(46.0 \%)$ for diarrhoea relief, and higher in the loperamide-simeticone caplet group $(79.1 \%)$ and chewable tablet group $(81.3 \%)$ than in the $S$. boulardii capsule group $(42.3 \%)$ for abdominal discomfort relief. 
Table 3 Study endpoints (intention-to-treat analysis, $n=415$ )

\begin{tabular}{|c|c|c|c|c|}
\hline Endpoints & $\begin{array}{l}\text { Loperamide- } \\
\text { simeticone caplet }\end{array}$ & $\begin{array}{l}\text { Loperamide-simeticone } \\
\text { chewable tablet }\end{array}$ & $\begin{array}{l}\text { Saccharomyces } \\
\text { boulardii capsule }\end{array}$ & Significance \\
\hline$N$ & 139 & 139 & 137 & \\
\hline $\begin{array}{l}\text { Number of unformed stools } 0-24 \mathrm{~h} \\
\text { (mean) }\end{array}$ & 3.4 & 3.3 & 4.3 & $\begin{array}{l}\text { One-sided } 97.5 \% \text { CI (tablets- } \\
\text { caplets): }-0.50 \text { to infinity } \\
\text { Two-sided } 95 \% \text { CI ( } S \text {. boulardii- } \\
\text { caplets): } 0.43-1.22 \\
\text { Two-sided } 95 \% \text { CI (S. boulardii- } \\
\text { tablets: } 0.55-1.34\end{array}$ \\
\hline $\begin{array}{l}\text { Time to last unformed stool [median } \\
\text { (h)] }\end{array}$ & 14.9 & 14.0 & 28.5 & $\begin{array}{l}\text { Caplet vs. tablet, } p=0.82 \\
\text { Caplet vs. } S \text {. } \text { boulardii, } p<0.001 \\
\text { Tablet vs. } S . \text { boulardii, } p<0.001\end{array}$ \\
\hline $\begin{array}{l}\text { Time to complete relief of diarrhoea } \\
\text { [median (h)] }\end{array}$ & 26.0 & 26.0 & 45.8 & $\begin{array}{l}\text { Caplet vs. tablet, } p=0.50 \\
\text { Caplet vs. } S \text {. } \text { boulardii, } p<0.001 \\
\text { Tablet vs. } S \text {. } \text { boulardii, } p<0.001\end{array}$ \\
\hline $\begin{array}{l}\text { Time to complete relief of abdominal } \\
\text { discomfort [median (h)] }\end{array}$ & 12.2 & 12.0 & 23.9 & $\begin{array}{l}\text { Caplet vs. tablet, } p=0.98 \\
\text { Caplet vs. } S . \text { boulardii, } p<0.005 \\
\text { Tablet vs. } S . \text { boulardii, } p<0.005\end{array}$ \\
\hline \multicolumn{5}{|c|}{ Subject's evaluation of treatment (\% very good or excellent) } \\
\hline Overall illness relief & 83.5 & 85.6 & 48.9 & $\begin{array}{l}\text { Caplet vs. tablet, } p=0.45 \\
\text { Caplet vs. } S \text {. } \text { boulardii, } p<0.001 \\
\text { Tablet vs. } S \text {. } \text { boulardii, } p<0.001\end{array}$ \\
\hline Diarrhoea relief & 79.9 & 84.9 & 46.0 & $\begin{array}{l}\text { Caplet vs. tablet, } p=0.12 \\
\text { Caplet vs. } S \text {. } \text { boulardii, } p<0.001 \\
\text { Tablet vs. } S . \text { boulardii, } p<0.001\end{array}$ \\
\hline Abdominal discomfort relief & 79.1 & 81.3 & 42.3 & $\begin{array}{l}\text { Caplet vs. tablet, } p=0.85 \\
\text { Caplet vs. } S \text {. } \text { boulardii, } p<0.001 \\
\text { Tablet vs. } S \text {. } \text { boulardii, } p<0.001\end{array}$ \\
\hline
\end{tabular}

CI confidence interval

The differences in the response frequencies between the loperamide-simeticone caplet or chewable tablet and $S$. boulardii capsule were statistically significant for all evaluations of treatment effectiveness $(p<0.001$ in each case); no significant difference was found between the loperamide-simeticone caplet and chewable tablet for any evaluation of treatment effectiveness.

\subsubsection{Follow-Up Endpoints}

Overall, most subjects $(95.3 \%)$ reported passing stool at least once since the final study visit, with similar proportions across the treatment groups; differences were not statistically significant (Table 4).

Most subjects $(94.3 \%)$ overall did not experience continued or recurrent diarrhoea after the final study visit. The proportion of subjects who reported either continuation or a return of diarrhoea since the final study visit was slightly higher in the $S$. boulardii capsule group (5.7\%) than in the loperamide-simeticone caplet group $(3.7 \%)$ and the loperamide-simeticone chewable tablet group (3.7\%). Differences between the loperamide-simeticone caplet and tablet and the loperamide-simeticone tablet and $S$. boulardii capsule were not significant, while the difference between the loperamide-simeticone caplet and $S$. boulardii capsule was significant $(p<0.03)$.

Most subjects reported feeling completely well at the follow-up evaluation (loperamide-simeticone caplets and tablets $96.3 \%$; S. boulardii capsules $88.6 \%$ ). The differences between both loperamide-simeticone groups and the $S$. boulardii capsule were statistically significant $(p<0.02)$.

\subsection{Tolerability}

A total of $17(4.1 \%)$ subjects experienced at least one adverse event; three $(2.2 \%)$ subjects in the loperamidesimeticone caplet group, seven $(5.0 \%)$ subjects in the loperamide-simeticone chewable tablet group and seven (5.1\%) subjects in the $S$. boulardii capsule group. The most commonly reported adverse events were nausea, 


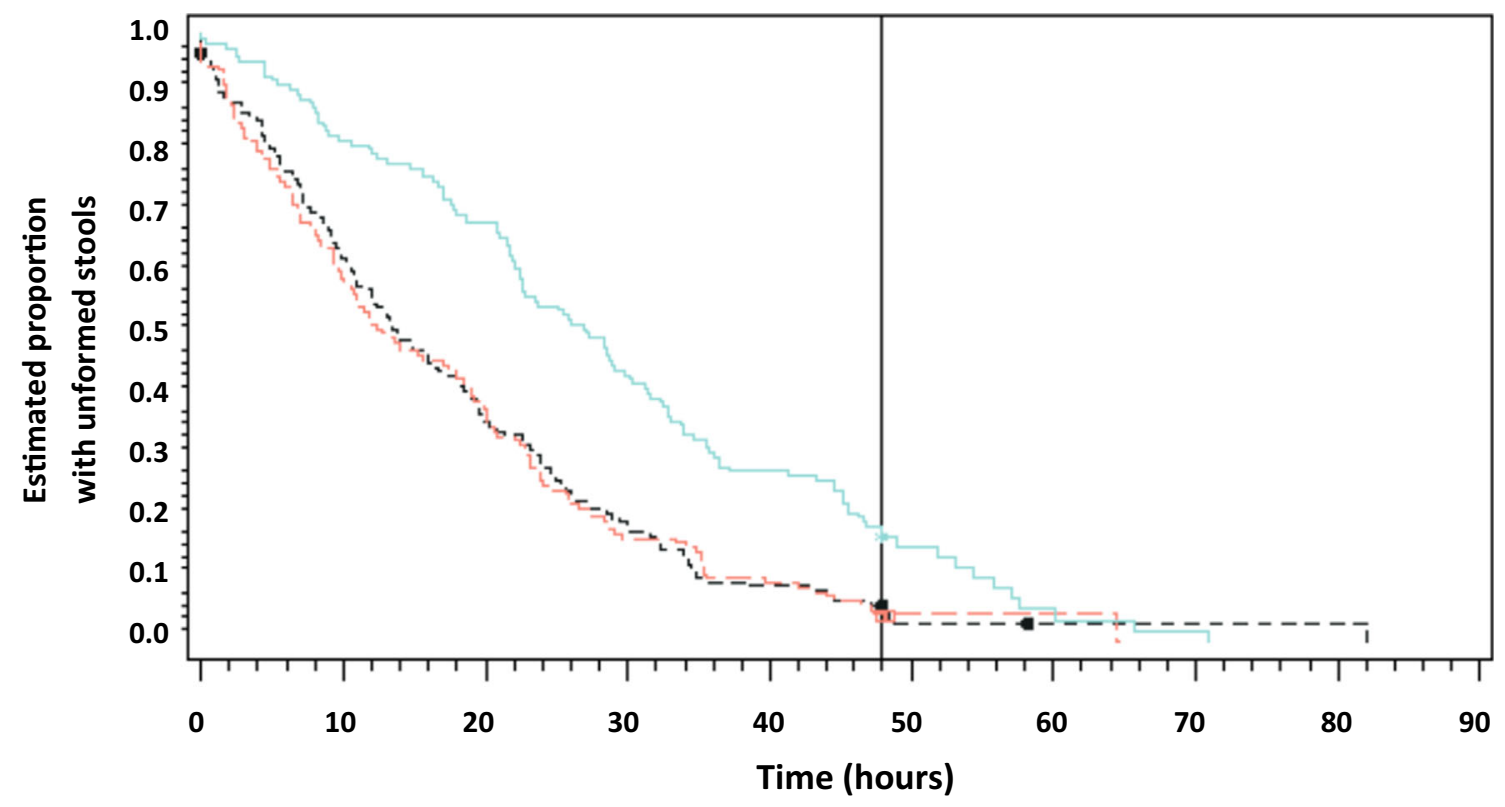

- - - - - Loperamide-simeticone caplet

- _ - - Loperamide-simeticone tablet

S. boulardii capsule

Fig. 2 Time to last unformed stool (Kaplan-Meier curve)

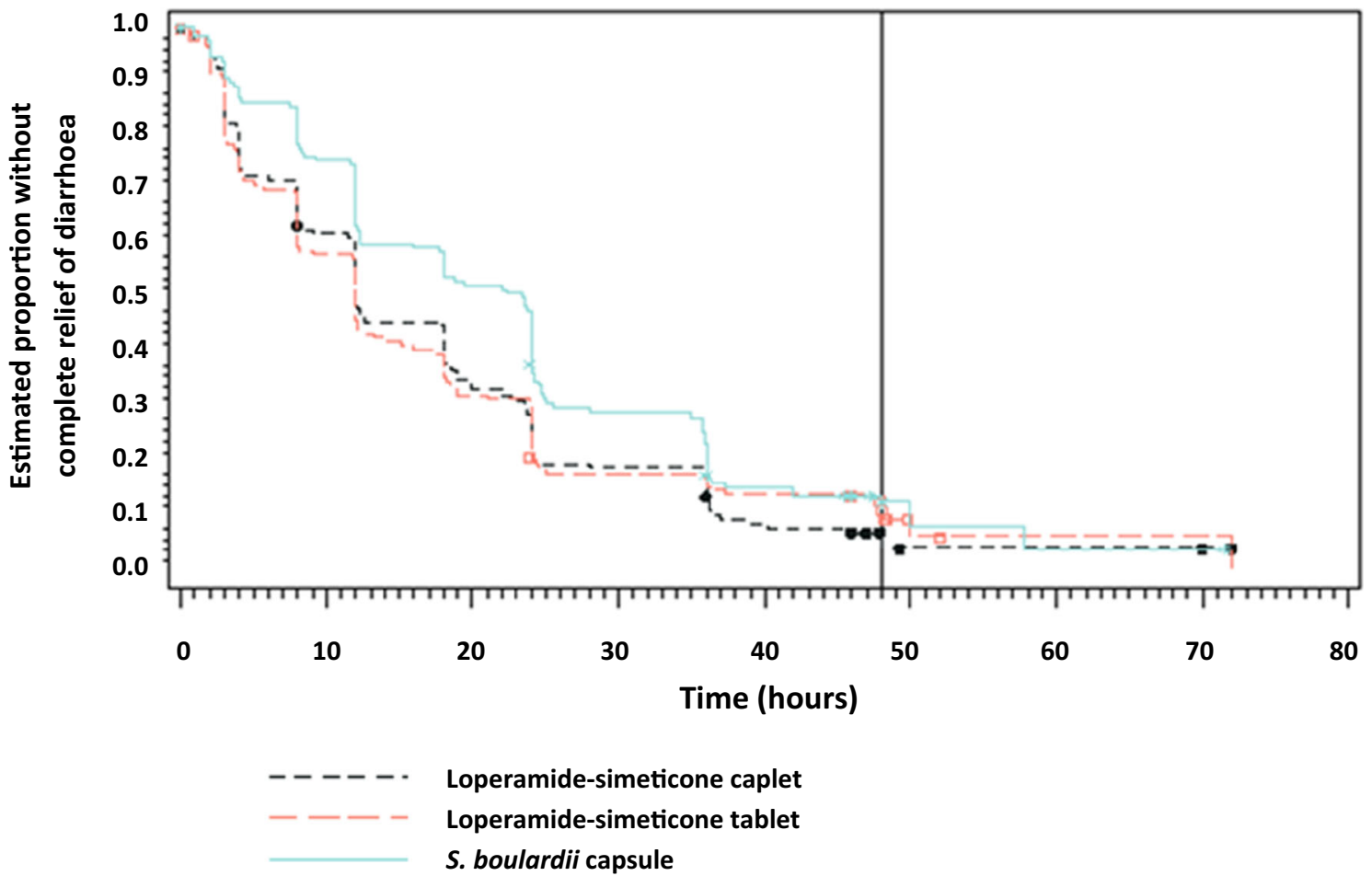

Fig. 3 Time to complete relief of diarrhoea (Kaplan-Meier curve)

asthenia and anorexia [in three $(0.7 \%)$ subjects each, overall]. Constipation was reported by two (1.4\%) subjects in the loperamide-simeticone chewable tablet group, with no reports in the other two groups. No subject experienced a serious adverse event or was withdrawn from the study due to an adverse event. 
Table 4 Follow-up evaluation at 7 days (all patients completed analysis, $N=403$ )

\begin{tabular}{|c|c|c|c|c|}
\hline Evaluation & $\begin{array}{l}\text { Loperamide- } \\
\text { simeticone } \\
\text { caplet }\end{array}$ & $\begin{array}{l}\text { Loperamide- } \\
\text { simeticone } \\
\text { chewable } \\
\text { tablet }\end{array}$ & $\begin{array}{l}\text { Saccharomyces } \\
\text { boulardii } \\
\text { capsules }\end{array}$ & Significance \\
\hline$N$ & 135 & 136 & 132 & \\
\hline $\begin{array}{l}\text { Passed stool }(\geq 1) \text { since } \\
\text { final study visit }(\%)\end{array}$ & 94.1 & 94.8 & 97.0 & $\begin{array}{l}\text { Caplet vs. tablet, } p=0.80 \\
\text { Caplet vs. } \text { S. boulardii, } \\
\quad p=0.25 \\
\text { Tablet vs. } \text { S. boulardii, } \\
\quad p=0.37\end{array}$ \\
\hline $\begin{array}{l}\text { Diarrhoea continued or } \\
\text { returned since final } \\
\text { study visit }(\%)\end{array}$ & 3.7 & 3.7 & 5.7 & $\begin{array}{l}\text { Caplet vs. tablet, } p=0.50 \\
\text { Caplet vs. } \text { S. } \text { boulardii, } \\
\quad p<0.03 \\
\text { Tablet vs. } \text { S. boulardii, } \\
p=0.11\end{array}$ \\
\hline $\begin{array}{l}\text { Feel completely well } \\
\text { following diarrhoeal } \\
\text { illness }(\%)\end{array}$ & 96.3 & 96.3 & 88.6 & $\begin{array}{l}\text { Caplet vs. tablet, } p=1.000 \\
\text { Caplet vs. } S . \text { boulardii, } \\
\quad p<0.02 \\
\text { Tablet vs. } \text { S. boulardii, } \\
p<0.02\end{array}$ \\
\hline
\end{tabular}

\section{Discussion}

This acute diarrhoea study was conducted in Mexico and Goa, India, areas with a high risk for acute diarrhoea, and included a mixed population of adult residents, expatriates and international travellers. TD is usually defined in studies as the passage of at least three unformed stools within a 24-h period, accompanied by at least one gastrointestinal symptom. Subjects in this study would meet this definition on clinical criteria, though by convention TD usually refers to acute diarrhoea that develops in a resident of the industrialised world who travels to a developing tropical or semitropical country. TD is most usually of bacterial origin, with enterotoxigenic Escherichia coli (ETEC) the major pathogen in most cases worldwide [22]. There are, however, geographical differences, with ETEC dominating in Latin America, while Campylobacter jejuni and Salmonella spp. are more important in Asian countries. Similar enteropathogens are also responsible for acute endemic diarrhoeas, although in residents the clinical course is milder [23]. In this study there was no attempt to identify a causative pathogen at the time of inclusion in the study. The treatments in this study were non-specific, and forms of clinical presentation that would have required further investigation (particularly persistent and dysenteric diarrhoea) were entry exclusions. Diagnostic yield is low using conventional laboratory methodologies, with no pathogen identified in up to half of cases [22]. Future studies may benefit from the use of PCR (polymerase chain reaction) diagnostic techniques for intestinal pathogens, which offers the advantages of increased sensitivity and greater timeliness of diagnosis [24]. Although the cause of acute diarrhoea was not determined in this study, $52 \%$ of the sample were international travellers to the study countries and it is probable on the basis of previous epidemiological studies that most cases were bacterial in origin.

Two previous double-blind, randomised studies demonstrated that the loperamide-simeticone chewable tablet was significantly more effective than either loperamide or simeticone alone or placebo over a 48-h study period $[8,9]$. This study has demonstrated that the caplet form of the loperamide-simeticone combination was noninferior in comparison with the original chewable tablet with respect to the primary endpoint of NUS 0-24, with means of 3.4 and 3.3 for the caplet and tablet, respectively. There were no statistically significant differences between the two formulations for any of the secondary endpoints, which supports the finding of non-inferiority for the primary endpoint. The two formulations show very similar efficacy and can therefore be considered clinically equivalent.

As a non-inferiority study, the design could be criticised for not including a placebo arm. However, guidelines [25] allow for an active-controlled non-inferiority design where there is sufficient demonstration of efficacy in well-conducted placebo-controlled studies $[8,9]$.

This study also compared the loperamide-simeticone combinations versus probiotic yeast capsules (S. boulardii). Previously no clinical trial has compared loperamide with a probiotic, and a formulation of $S$. boulardii capsule with a licensed indication for treatment of acute diarrhoea was selected for this study. 
The effect of $S$. boulardii has been investigated in the treatment and prevention of various forms of diarrhoeal diseases, with evidence of efficacy particularly in children and in more persistent diarrhoea, usually with an endpoint of reduced duration [12-21]. Although a meta-analysis of studies showed evidence of efficacy for $S$. boulardii in the prevention of TD [15], evidence for efficacy in treatment is lacking. There appears to be only one published study [26] investigating treatment of TD, in which $S$. boulardii was used to treat travellers in Tunisia. Subjects with TD were randomised to $600 \mathrm{mg}$ of $S$. boulardii $\left(1 \times 10^{10} /\right.$ day $)$ for 5 days or an active control treatment. Of 60 subjects enrolled, 43 completed the trial, but the duration of diarrhoea was not significantly different between those given S. boulardii (2.1 days) and those given ethacridine lactate and tannalbuminate (1.4 days). In our study, the S. boulardii capsule used at the approved dose of $250 \mathrm{mg}$ twice daily was significantly less effective than the loperamide-simeticone formulations for all primary and secondary endpoints. This suggests that there may be more of a role for S. boulardii in diarrhoea in children, more persistent diarrhoea or in prevention than in treatment of acute diarrhoea in adults.

Previous studies with loperamide-simeticone $[8,9]$ have not followed subjects beyond $48 \mathrm{~h}$ after the initial dose. In this study, follow-up at 7 days was obtained remotely. As expected for a self-limiting condition, most subjects felt completely well following their diarrhoeal illness, though the proportions were significantly higher with both loperamide-simeticone formulations than with the $S$. boulardii capsule. Continued or recurrent diarrhoea was reported by a small minority of subjects in each group; slightly fewer with the loperamide-simeticone formulations than with the S. boulardii capsule. At 7-day follow-up more than $94 \%$ of subjects reported passing stool at least once since the second study visit (i.e. between 4 and 5 days after visit 2), with no significant differences between the groups. In addition, there were two adverse event reports of constipation, both of which were in the loperamide-simeticone chewable tablet group (1.4\%). This supports findings from other well-controlled double-blind trials of acute diarrhoea that normal dosing schedules of loperamide are not associated with a significant incidence of constipation or constipation-like episodes.

\section{Conclusions}

In this study, the loperamide-simeticone caplet formulation was demonstrated to be non-inferior to the original chewable tablet formulation; both formulations can be expected to demonstrate similar clinical efficacy in the relief of symptoms of acute diarrhoea. Both formulations of loperamide-simeticone were consistently superior to the $S$. boulardii capsule with regards to the primary and secondary endpoints.

Acknowledgments Loperamide-Simeticone Study Team: Mexico J. E. R. Orozco MD, H. O. E. Picazo MD (Puerto Vallarta, Jalisco), R. Maxwell MD (Guanaujuato), E. M. Espino MD (San José del Cabo Baja California Sur); India D. Naik MD (Mapusa, Goa), V. Naik MD (Dona Paula, Goa).

The authors would like to thank Ulla Lantz, McNeil AB, Helsingborg, Sweden, for project management support throughout the study

\section{Compliance with Ethical Standards}

Ethical standards This study was conducted in accordance with the principles of the 1964 Declaration of Helsinki and later amendments, and with applicable Good Clinical Practice (GCP) guidelines. Local regulatory requirements were followed, and the study protocol and associated documents were reviewed and approved by the Ethics Committees for each participating site. The study was registered on ClinicalTrials.gov (NCT00807326). All subjects provided written informed consent prior to inclusion in the study.

Declaration of funding This study was sponsored by McNeil AB, a Johnson \& Johnson Company.

Declaration of financial/other relationships JC, RP and RH were employees of Johnson \& Johnson companies at the time the study was conducted.

Open Access This article is distributed under the terms of the Creative Commons Attribution-NonCommercial 4.0 International License (http://creativecommons.org/licenses/by-nc/4.0/), which permits any noncommercial use, distribution, and reproduction in any medium, provided you give appropriate credit to the original author(s) and the source, provide a link to the Creative Commons license, and indicate if changes were made.

\section{References}

1. Manatsathit S, DuPont HL, Farthing M, et al. Working Party of the Program Committee of the Bangkok World Congress of Gastroenterology 2002. Guideline for the management of acute diarrhea in adults. J Gastroenterol Hepatol. 2002;17(Suppl):S54-71.

2. von Sonnenburg F, Tomieporth N, Waiyaki P, et al. Risk and aetiology of diarrhoea at various tourist destinations. Lancet. 2000;356:133-4.

3. Steffen R. Epidemiology of traveler's diarrhea. Clin Infect Dis. 2005;41(suppl 8):S536-40.

4. Wingate D, Phillips SF, Lewis SJ, et al. Guidelines for adults on self-medication for the treatment of acute diarrhoea. Aliment Pharmacol Ther. 2001;15:773-82.

5. DuPont HL, Ericsson CD, Farthing MJG, et al. Expert review of the evidence base for self-therapy of travelers' diarrhea. J Travel Med. 2009;16:161-71.

6. Kennedy J. Self care of acute diarrhoea in non-travelling adults. SelfCare. 2010;1:50-3.

7. Farthing M, Salam M, Lindberg G, et al. Acute diarrhea in adults and children: a global perspective. J Clin Gastroenterol. 2013;47:12-20.

8. Kaplan MA, Prior MJ, Ash RR, et al. Loperamide-simethicone vs loperamide alone, simethicone alone, and placebo in the 
treatment of acute diarrhea with gas-related abdominal discomfort. Arch Fam Med. 1999;8:243-8.

9. Hanauer SB, DuPont HL, Cooper KM, et al. Randomized, double-blind, placebo controlled clinical trial of loperamide plus simethicone vs. loperamide alone and simethicone alone in the treatment of acute diarrhea with gas-related abdominal discomfort. Curr Med Res Opin. 2007;23:1033-43.

10. Connor AL, Wray H, Cottrell J, et al. A scintigraphic study to investigate the potential for altered gut distribution of loperamide from a loperamide-simethicone formulation in man. Eur J Pharm Sci. 2001;13:369-74.

11. Placidi E, Marciani L, Hoad CL, et al. The effects of loperamide, or loperamide plus simethicone, on the distribution of gut water as assessed by MRI in a mannitol model of secretory diarrhoea. Aliment Pharmacol Ther. 2012;36:64-73.

12. Czerucka D, Piche T, Rampal P. Review article: yeast as probiotics-Saccharomyces boulardii. Aliment Pharmacol Ther. 2007;26:767-78.

13. Szajewska H, Skorka A, Dylag M. Meta-analysis: Saccharomyces boulardii for treating acute diarrhoea in children. Aliment Pharmacol Ther. 2007;25:257-64.

14. Allen SJ, Martinez EG, Gregorio GV, et al. Probiotics for treating acute infectious diarrhoea. Cochrane Database Syst Rev 2010;10:CD003048.

15. McFarland LV. Systematic review and meta-analysis of Saccharomyces boulardii in adult patients. World J Gastroenterol. 2010;16(18):2202-22.

16. Keseledis T, Pothoulakis C. Efficacy and safety of the probiotic Saccharomyces boulardii for the prevention and therapy of gastrointestinal disorders. Ther Adv Gastroenterol. 2012;5(2):111-25.

17. Guarino A, Dupont C, Gorelov AV, et al. The management of acute diarrhea in children in developed and developing areas: from evidence base to clinical practice. Exp Opin Pharmacother. 2012;13(1):17-26.

18. Dinleyici EC, Eren M, Ozen M, et al. Effectiveness and safety of Saccharomyces boulardii for acute infectious diarrhea. Exp Opin Biol Ther. 2012;13(1):17-26.

19. Ritchie ML, Romanuk TN. A meta-analysis of probiotic efficacy for gastrointestinal diseases. PLoS One. 2012;7(4):e34938.

20. Feizizadeh S, Amin Salehi-Abargouei A, Vajihe Akbari V. Efficacy and safety of Saccharomyces boulardii for acute diarrhea. Pediatrics. 2014;134:e176-91.

21. Passariello A, Agricole P, Malfertheiner P. A critical appraisal of probiotics (as drugs or food supplements) in gastrointestinal diseases. Curr Med Res Opin. 2014;30(6):1055-64.

22. Peltola H, Gorbach SL. Travelers' diarrhea. In: DuPont HL, Steffen R, editors. Textbook of travel medicine and health. Hamilton: B C Decker Inc.; 2001.

23. Bouckenooghe AR, Jiang ZD, de la Cabada FJ, et al. Enterotoxigenic Escherichia coli as cause of diarrhea among Mexican adults and US travelers in Mexico. J Travel Med. 2002;9:137-40.

24. Spina A, Kerr KG, Cormican M, et al. Spectrum of enteropathogens detected by the FilmArray GI Panel in a multicentre study of community-acquired gastroenteritis. Clin Microbiol Infect. 2015;21:719-28.

25. European Medicines Agency. ICH Topic E 10: choice of control group in clinical trials. Note for guidance on choice of control group in clinical trials (CPMP/ICH/364/96) January 2001. http:// www.ema.europa.eu/docs/en_GB/document_library/Scientific_ guideline/2009/09/WC500002925.pdf. Accessed 5 Oct 2015.

26. Bruns R, Raedsch R. Therapy of traveller's diarrhea [in German]. Med Welt. 1995;46:591-6. 